\title{
Allosteric Networks Governing Regulation and Catalysis of Src-Family Protein Tyrosine Kinases - Implications for Disease-Associated Kinases
}

Heung-Chin Cheng, ${ }^{l \neq}$ Timothy M. Johnson ${ }^{1,2}$, Ryan D. Mills, ${ }^{1,2}$ Yuh-Ping Chong, ${ }^{2}$ Khai-Chew Chan, ${ }^{1}$ and Janetta G. Culvenor, ${ }^{2}$

${ }^{1}$ Department of Biochemistry and Molecular Biology, Bio21 Molecular Science and Biotechnology Institute, and ${ }^{2}$ Department of Pathology, University of Melbourne, Parkville, Victoria 3010, Australia

₹ Address all correspondence to:

Dr. Heung-Chin Cheng

Department of Biochemistry and Molecular Biology

Bio21 Institute

University of Melbourne

30 Flemington Road

Parkville, Victoria 3010

$\mathrm{Ph}$ : International (613)-83442254

Fax: (613)-93481421

E-mail: heung@unimelb.edu.au

Short Title: Allosteric Regulation and Catalysis of Src-Family Protein Kinases

Key words: Protein tyrosine kinases, Oncogene, Phosphotransferase, Cancer, Inhibitors. SrcFamily Kinases, and Protooncogene Proteins pp60 $0^{\mathrm{C}-\mathrm{Src}}$ 


\section{Summary}

The Src-family protein tyrosine kinases (SFKs) are multi-domain oncogenic protein tyrosine kinases. Over-activation of SFKs contributes to cancer formation and progression. Thus, synthetic inhibitors of SFKs are being developed as therapeutics for cancer treatment. Understanding the regulatory and catalytic mechanisms of SFKs is necessary for the development of therapeutic SFK inhibitors. Although many upstream regulators and protein substrates of SFKs have been identified, both the mechanisms of activation and catalysis of SFKs are not fully understood. In particular, it is still unclear how the inactive SFKs undergo conformational transition during activation. The mechanism governing the binding of substrates and the release of products during catalysis is another area that requires investigation. Several recent publications indicate the presence of a "hydrophobic spine" formed by four conserved interacting hydrophobic residues in the kinase domain of SFKs. In this manuscript, we discuss how the assembly and disassembly of the hydrophobic spine residues may govern conformational transition of SFKs during activation. In addition to regulation of kinase activity, the hydrophobic spine is implicated to be involved in catalysis. A recent manuscript postulates that perturbation of the hydrophobic spine residues is a key step in catalysis (Shan et al. Proc. Natl. Acad. Sci., 106, 139-144). Further investigations to decipher the roles of the hydrophobic spine residues in regulation and catalysis of SFKs will benefit the development of therapeutic SFK inhibitors for cancer treatment.

\section{(228 words)}


The Src-family tyrosine kinases (SFKs) are a group of ubiquitously expressed protein kinases involved in a wide spectrum of cellular functions ranging from cell migration to ion channel activity of neurotransmitter receptors (review in (1)). Aberrant activation and expression of Src-family kinases contribute to pathogenesis and progression of many diseases. For example, over-activation and overexpression of the SFK members Lyn and Fyn are involved in the development of drug-resistant chronic myelogenous leukaemia $(2,3)$; activation of another SFK member Hck by the type 1 human immunodeficiency virus (HIV-1) accessory protein Nef has been implicated to contribute to the progression of the disease from infection to AIDS (4). Owing to the significant role played by SFKs in disease, synthetic inhibitors of SFKs are potential therapeutics for disease treatment. This manuscript aims to give an overview of the current state of knowledge of the structural basis of the conformational transition governing regulation of the kinase activity and catalysis of SFKs. Several groups of researchers reported the existence of a regulatory hydrophobic spine that appropriately organizes the catalytically critical amino acid residues for the phospho-transfer reaction. Configurational changes of the hydrophobic spine have been implicated to play an important role in catalysis in a related protein tyrosine kinase c-Abl. The possibility that similar configurational changes also apply to SFK catalysis is discussed. The crystal structure of a SFK member c-Src complexed with the upstream regulatory kinase CSK has been solved. How this structure contributes to our understanding of the structural basis of inhibition of SFKs by the two upstream regulatory kinases CSK and CHK is another focus of this manuscript. 


\section{Overview of SFK structure}

SFKs are multi-domain enzymes containing the kinase domain and several functional domains directing protein-protein interactions and interaction of SFKs with plasma membrane. These non-catalytic domains govern substrate recognition and subcellular localization of SFKs. In addition to binding other cellular proteins, the additional domains also interact intramolecularly to control the activity of the kinase domain. In normal non-proliferating cells, SFKs are kept mostly in the inactive state by two endogenous upstream inhibitory kinases, Cterminal Src kinase (CSK) and its homolog CHK. These two kinases inhibit SFKs by specifically phosphorylating a consensus regulatory tyrosine near the $\mathrm{C}$-terminus (referred to as $\mathrm{Y}_{\mathrm{T}}$ ). Upon phosphorylation at $\mathrm{Y}_{\mathrm{T}}$, SFKs have a high propensity to adopt the inactive conformation. As shown in Fig. 1A, SFKs are kept in the inactive conformation by two inhibitory intramolecular interactions: (i) the $\mathrm{pY}_{\mathrm{T}}-\mathrm{SH} 2$ interaction involving binding of the phosphorylated $\mathrm{Y}_{\mathrm{T}}\left(\mathrm{p} \mathrm{Y}_{\mathrm{T}}\right)$ to the SH2 domain, and (ii) the linker-SH3 interaction in which the SH2-kinase linker binds the SH3 domain $(5,6)$. Several manuscripts published by Roux and co-workers in recent years describe their study of the pathways by which SFKs in the inactive conformation transits to the active conformation by molecular dynamics simulation $(7,8)$. In this manuscript, we review their findings in the context of the experimental results obtained by our group and other researchers.

The kinase domain of the SFK member c-Src consists of 256 residues. It can be subdivided into the small (residues 264-331) and large (residues 332-519) lobes. $\mathrm{Mg}^{++}-\mathrm{ATP}^{\mathrm{T}}$ binds to a cleft surrounded by the glycine-rich loop (residues 274-279) of the small lobe, and the catalytic loop containing the catalytic base Asp-386. The proper orientation of ATP is governed by (i) the glycine rich loop (G-loop) which interacts with the ATP $\beta$-phosphate group, (ii) invariant lysine (Lys-295) binds the $\alpha$ - and $\beta$-phosphates of ATP and (iii) the $\mathrm{Mg}^{++}$ion that 
chelates the $\beta$ - and $\gamma$-phosphates of ATP. Proper positioning of Lys-295 is regulated by a salt bridge formed between its side chain and the carboxyl side chain of Glu-310 in the C-helix. The position of the $\mathrm{Mg}^{++}$ion is stabilised by binding to the deprotonated carboxyl side chain of Asp404 of the activation loop (A-loop, residues 404-432) (Fig. 1B). Catalysis of the phosphorylation reaction requires participation of a catalytic base (Asp-386 of the catalytic loop) (9). During catalysis, the Asp-386 side chain interacts with the hydroxyl group of the target tyrosine of the protein substrate, facilitating the transfer of $\gamma$-phosphate of ATP to the target tyrosine. The consensus Tyr-416 in the A-loop can undergo autophosphorylation. Electrostatic interactions formed between phospho-Tyr-416 (pY416) and Arg-409 further stabilize the active conformation of c-Src kinase domain.

In the inactive conformation stabilized by the two inhibitory intramolecular interactions, the catalytically critical residues are aligned in a pattern that is not suitable for catalysis (Fig. 1C). Specifically, the C-helix undergoes rotation such that Glu-310 is pointing away from the active site; therefore, it is unable to form a salt bridge with Lys-295. Consequently, Lys-295 is unable to position properly to bind ATP. As the C-helix and A-loop are coupled, rotation of the C-helix forces the A-loop to adopt a configuration that occludes the active site, preventing the binding of protein substrates.

\section{The hydrophobic spine properly organizes the catalytically critical residues of the kinase domain to catalyse the phosphorylation reaction}

In the past few years, several groups of researchers reported the existence of a hydrophobic spine in the kinase domain that governs the proper alignment of the catalytically 
critical residues $(10,11)$. By comparison of the distribution patterns of residues of the kinase domain in the active and inactive conformations of many protein kinases, Kornev et al. $(10,12)$ discovered that four conserved hydrophobic residues in the kinase domain are assembled into a "spine-like" formation (hence the name "hydrophobic spine") in the active conformation of protein kinases (Fig. 1B). The hydrophobic spine is disassembled when the kinases adopt the inactive conformations (Fig. 1C). Relevant to this, Bukhtiyarova, et al. reported that mutation of one of the hydrophobic spine residue (Phe-185 of the A-loop) to alanine, arginine or glycine completely abolishes the catalytic activity of the p38 MAP kinase (13).

In c-Src, the four conserved residues making up the hydrophobic spine are: Leu-325 of the 34 -strand, Met-314 of the C-helix, Phe-405 of the A-loop and His-384 of the catalytic loop (14). When these four residues are assembled in the spine-like formation, all the catalytically critical residues are appropriately aligned for the phospho-transfer reaction. In SFKs, the SH2kinase linker acts as a switch that controls the inter-conversion of the active and inactive conformation of the kinase domain. Trp-260 is the key residue in the linker interacting with the kinase domain and in turn governing the inter-conversion. Leu-325 of the hydrophobic spine is one of the hydrophobic residues in the kinase domain interacting with Trp-260. In the hydrophobic spine, the four hydrophobic residues are stacked in such a way that configurational changes of the linker are transmitted via Trp-260 to various motifs of the kinase domain. The pathway of transmission of configurational changes in the linker follows the pattern of W260 to L325, L325 to M314 of the C-helix, M314 to F405 of the A-loop. The changes are finally transmitted from F405 to H384 of the catalytic loop (Fig. 1B).

In addition to interaction with Trp-260 of the SH2-kinase linker, Leu-235 of the hydrophobic spine also interacts with Thr-338, the conserved "gatekeeper" residue which binds 
the adenine moiety of ATP and small molecule inhibitors. It is likely that the L325-T338 interaction influences the assembly of the hydrophobic spine and this in turn controls the kinase activity of c-Src (Fig. 1B). Relevant to this, mutation of Thr-338 to Ile was found to induce significant activation of c-Src (14) (Fig. 1B). Furthermore, mutation of the gatekeeper threonine residue to a hydrophobic residue also induces significant activation of several other tyrosine kinases including c-Abl, PDGF-receptor $\alpha$ and $\beta$, and EGF-receptor (14). Intuitively, the conversion of the gatekeeper threonine to isoleucine is expected to strengthen the hydrophobic interaction with Leu-325. It is likely that the strengthened L325-I338 interaction enhances assembly of the hydrophobic spine in the c-Src mutant. More importantly, the results suggest that mutation of the gatekeeper threonine to a hydrophobic residue is a common mechanism of activation of tyrosine kinases (14).

\section{Configurational changes of the SH2-kinase linker govern the conformational transition of SFK kinase domain}

Since the kinase domain of SFK is constitutively active (15), it needs to interact with other structural motifs to constrain itself in the inactive conformation. The SH2-kinase linker (referred to as the linker) plays the most important role in constraining the kinase domain in the inactive conformation. In the presence of the inhibitory $\mathrm{pY}_{\mathrm{T}}-\mathrm{SH} 2$ and linker-SH3 interactions, Trp-260 of the linker inserts between the C-helix and $\beta 4$ strand in the small lobe of the kinase domain (Fig. 2B). The Trp-260-binding pocket is formed by Phe-307 and Leu-308 of the Chelix, and Leu-325, Tyr-326, Ala-327 and Val-328 of the $\beta 4$ strand. These interactions force the C-helix to rotate such that Glu-310 is pointing away from the active site. 
Disruption of the $\mathrm{pY}_{\mathrm{T}}-\mathrm{SH} 2$ and/or linker-SH3 interactions allows the linker to adopt a different configuration, removing the constraints it imposes on the kinase domain (16-18). In this configuration, Trp-260 no longer interacts with Phe-307 and Leu-308 of the C-helix (Fig. 2C). Consequently, the C-helix undergoes inward rotation such that the side chain of Glu-310 points towards the active site and forms salt bridge with Lys-295. In addition, the linker in this configuration participates in stabilizing the active conformation of the kinase domain. Arg-264, which is contiguous with the linker forms electrostatic interactions with Glu-331 and Pro-333 of the loop between the $\beta 4$ - and $\beta 5$-strands ( $\beta 4.5$-loop) (Fig. 2 C). Huang et al. found that the R264$\beta 4.5$-loop interaction stabilizes the SFK kinase domain in the active conformation (15).

\section{The conformational transition pathway of activation of SFKs}

Using molecular dynamics simulation, Roux and co-workers mapped the pathway of conformational transition leading to activation of SFKs (7). Remodeling of two major hubs of intramolecular interactions governs the transition: (i) W260-C-helix interactions and (ii) C-helixA-loop interactions. The simulation also identified two intermediate states in the pathways $\left(\mathrm{I}_{1}\right.$ and $\mathrm{I}_{2}$ in Fig. 2A). Transition of the inactive conformation to $\mathrm{I}_{1}$ involves abolition of the inhibitory W260-C-helix interaction (Trp-260 interacting with Phe-307 and Leu-308). Its abolition allows disruption of the hydrophobic cluster formed by Phe-307 and Met-314 of the Chelix and Leu-407 of the A-loop during transition from $I_{1}$ to $I_{2}$ of the pathway. In $I_{2}$, the C-helix begins to rotate inward. The E310-K295 salt bridge formation is formed when $\mathrm{I}_{2}$ is converted to the active conformation. Their simulation estimated that the transition from inactive to active conformations lasts for $0.1-0.2 \mu \mathrm{sec}$. The distribution of the four states of SFKs was estimated 
to be: inactive conformation (57\%), $\mathrm{I}_{1}(20 \%), \mathrm{I}_{2}(14 \%)$ and active conformation (9\%). The high abundance of the inactive conformation is favored by the $\mathrm{pY}_{\mathrm{T}}-\mathrm{SH} 2$ and linker-SH3 interactions. Conceivably, disruption of these interactions by ligands of the SH2 and SH3 domains are expected to alter the distribution in favor of the active conformation.

In summary, biochemical, structural and molecular dynamics simulation studies indicate that the linker, C-helix and A-loop are coupled in specific patterns in both the active and inactive conformations of SFKs. Transition of SFKs from the active to the inactive conformations involves transient uncoupling and re-organisation of the coupling patterns. Furthermore, even in the absence of ligands that target the $\mathrm{SH} 2$ and $\mathrm{SH} 3$ domain, the kinase domain in the inactive conformation of SFKs possesses the intrinsic ability to undergo transition to the active conformation.

\section{The DFG-flip and the catalytic cycle of protein kinases}

The structural determination of various protein kinase domain conformations has provided significant insight into the mechanism of protein kinase-mediated catalysis of $\gamma$ phosphate transfer from ATP to substrate. Recent crystal structures of c-Abl and c-Src bound to the ATP analogue imatinib and variants engineered to bind equally well to both kinases indicate that the crystallised forms assume conformations in which the aspartate of the conserved DFGmotif is disengaged from the active site of the kinase domain thereby rendering it ineffective with regard to coordination of $\mathrm{Mg}^{2+}$-ATP (19). Furthermore, c-Src bound to a derivative of PPI also adopt a similar conformation (20). The structures indicate that the aspartate and phenylalanine residues have swapped positions in an apparent flip of the DFG conformation (Fig 
3). Notably, what seems at first appearance to assume an inactive conformation due to the inability to coordinate $\mathrm{Mg}^{2+}$-ATP, is that the kinase domain otherwise maintains an active conformation, with the $\mathrm{C}$-helix rotated inward so as to correctly align the catalytic residues in a conformation conducive for catalysis and the hydrophobic spine of the N-terminal lobe remaining in an active 'assembled' conformation (bottom right panel of Fig. 3). Additionally, molecular dynamics simulations have indicated that this DFG-Asp-out flip is promoted through the protonation of the aspartate residue (21). This renders it energetically more favorable to occupy the hydrophobic environment in which it finds itself following the flip out of the active site. Although the C-helix is in the active 'in' conformation in the both the imatinib-bound DFGAsp-out and the DFG-Asp-in structures of Abl kinase, molecular dynamics simulation indicates that an intermediate form in which the $\alpha$-C-helix moves to an inactive 'out' form may occur as part of the DFG flip (21). Therefore, the DFG-flip appears through simulation to be coupled to an $\alpha$-C-helix rotation to an 'out' conformation which also facilitates the formation of a hydrophobic pocket at the base of the N-terminal lobe for the DFG-phenylalanine to occupy prior to flipping into the ATP-binding site.

These findings have allowed for the proposal of a model for the catalytic cycle of a kinase domain (Fig 3). Upon binding of substrate to the DFG-Asp-in conformation, phosphorylation may occur, as the DFG-aspartate is in the correct alignment for $\mathrm{Mg}^{2+}$-ATP coordination. The aspartate residue remains deprotonated at this stage, conducive to the coordination of the $\mathrm{Mg}^{2+}$ ion. Following transfer of the $\gamma$-phosphate of ATP to substrate, MgADP and the phosphorylated substrate are released to enable binding of new substrate and MgATP. $\mathrm{Mg}^{2+}$ release following substrate phosphorylation is proposed to result in a change in the electrostatic environment, conducive to protonation of the DFG-aspartate, thereby increasing the 
favourability of the outward flip of the DFG-aspartate and removal of ADP from the active site. Following product dissociation, the activation loop can reassume the DFG-Asp-in conformation, where upon re-binding of $\mathrm{Mg}^{2+}$-ATP the electrostatic environment would promote its deprotonation through mediating an increase in its $\mathrm{pK}_{\mathrm{A}}$ and facilitating $\mathrm{Mg}^{2+}$-ATP recoordination. With the binding of a new substrate molecule, the kinase would be ready for another round of catalysis. This model indicates that the DFG motif of protein kinases may be universally employed, in conjunction with a coupled C-helix rotation to drive a continuous catalytic cycle. Furthermore, the rate at which the DFG-aspartate flips in and out of the active site is likely to be a factor in determining the maximal turnover rate of a given kinase domain, so helping to explain the variability in turnover rate between different kinase domains. The rate at which the DFG-motif flips may in turn be determined by the level of interaction made between the activation loop and the hydrophobic spine. Evidence for modulation of the conformational freedom of the activation loop by its interaction with the hydrophobic spine is provided by the finding that mutation of the gatekeeper glutamine residue of Erk2 to smaller glycine or alanine residues, results in greater activation loop flexibility, thereby allowing for intramolecular autophosphorylation and activation to occur in the absence of activating kinase (11). In this case, the more conformationally restrictive interaction of the activation loop with the hydrophobic spine is disrupted through the gatekeeper residue mutation.

\section{CSK and CHK Inhibition of Src-family Kinases}

CSK and CHK are the two most important endogenous inhibitors of SFKs identified thus far (22). Both CSK and CHK can inactivate Src-family kinases by exclusively phosphorylating the C-terminal inhibitory tyrosine residue $\mathrm{Y}_{\mathrm{T}}$ (Tyr-527 of the chicken c-Src sequence) in the C- 
terminal tail of Src. In addition to this phosphorylation dependent inactivation mechanism, we previously reported that CHK possesses a unique non-catalytic Src inhibitory mechanism - CHK directly binds Src-family kinases, the binding alone is sufficient to suppress Src autophosphorylation and kinase activity $(23,24)$. Interestingly, this non-catalytic inhibitory mechanism is only applicable to CHK, albeit the homologue CSK is highly similar to CHK (both proteins share $\sim 90 \%$ sequence identity in their kinase domains) (23, 24). Based upon these findings, this Section compares the mechanistic similarities and differences employed by CSK and CHK to inhibit Src-family kinase activity.

The molecular features of CSK recognition of SFKs have been characterised by several groups. Using a peptide library screening approach, early evidence shows that short peptides harbouring Tyr-527 derived from the C-terminal tail of SFKs, cannot be efficiently phosphorylated by CSK (25). This observation led to the conclusion that effective Src phosphorylation by CSK is not only limited to the local structure near Tyr-527 of Src, rather, it requires other binding motifs distant to its $\mathrm{C}$-terminal tail. In line with this notion, further work shows that a specific region in the CSK kinase domain corresponding to the $\alpha$-helix-D (residues RSRGRS-) mediates the long-range interaction between CSK and Src-family kinases (Fig. 4A). This docking motif allows CSK to bind and orients Tyr-527 into its active site for C-terminal tail phosphorylation (26). Intriguingly, mutations at the critical amino acid residues in the $\alpha$-helix-D only disrupt the CSK:Src binding, but not the catalytic activity of CSK. To provide further insights into this enzyme-substrate complex, it was speculated that Glu-510, Tyr-511, Asp-518 of Src and Arg-279, Arg-281, Arg-283 of CSK play important roles in stabilising the CSK:Src intermolecular interaction by forming the essential salt bridges (27). These observations were 
confirmed by the recently solved crystal structure of a complex between the kinase domains of CSK and c-Src, as illustrated in Fig. 4 (28).

The CSK:c-Src crystal structure (Fig. 4B) has shed some light on the structural basis of Src-family kinase inactivation by CSK. The structure indicates that CSK:c-Src intimate binding is important for positioning the C-terminal tail of c-Src in the vicinity of CSK active site, in order to permit substrate phosphorylation of Tyr-527 of c-Src. The structure also provides information that a helical 'kink,' formed by Tyr-519 and Phe-520 near the junction of $\alpha$-helix I and Cterminal tail of c-Src, fits well into a docking groove beneath the active site of CSK (Fig. 4B). Close inspection of the structure reveals that the Src C-terminal tail is disengaged, but interestingly it is not directly targeted into the CSK active site, suggesting further conformational changes of CSK in the complex are required to complete the phosphorylation of Tyr-527.

\section{Implications of the findings of c-Src/CSK complex to the novel non-catalytic inhibitory mechanism of CHK}

We previously demonstrated that CHK can employ a novel non-catalytic inhibitory mechanism to suppress the activity of c-Src and several other SFKs. We demonstrated that CHK can form a stable complex with SFKs; the CHK-SFK binding is a prerequisite for inhibiting all active forms of SFKs by suppressing their autophosphorylation, regardless of the phosphorylation status of Tyr-527 $(23,24)$. CHK only binds SFKs with their two inhibitory intramolecular interactions disrupted. However, it does not form a complex with SFKs in the inactive conformation. Relevant to our finding, Levinson et al. (28) also found that CSK does not bind $\mathrm{c}-\mathrm{Src}$ in the inactive conformation. 
Fig. 4A shows that the basic residues (Arg-279, Arg-281, Arg-283 of the $\alpha$-D helix, and Arg-384 and Arg-389 of the $\alpha \mathrm{F}-\alpha \mathrm{G}$ loop) essential for CSK/c-Src complex formation are conserved in CSK and CHK. It is therefore logical to predict the homologous basic residues in CHK (i.e. Arg-276, Arg-278, Arg-280, Arg-384 and Lys-389) are involved in mediating the CHK-SFK complex formation. Since the binding of CHK to SFKs is much tighter than CSKSFK binding (24), in addition to these basic residues, CHK likely employs other residues to strengthen its interaction with SFKs.

How might CHK binding suppress SFK activity? One possible explanation is that the binding of CHK to SFK kinase domain perturbs the alignment of catalytically critical residues in SFKs. Relevant to this, the kinase domain of c-Src complexed with CSK adopts a conformation distinct from the active (DFG-Asp-in), DFG-Asp-out, and inactive conformations of c-Src (Fig. 4D). The C-helix rotates outward such that the Glu-310 side chain points outside the active site. In this orientation, Glu-310 is unable to form a salt-bridge with Lys-295. Thus, c-Src in this distinct conformation is inactive.

\section{Implications for other protein kinases I - the effect of mutations of DFG on kinase activity}

Understanding of the function of the DFG motif in mediating enzymatic turnover will be important not only for the generic understanding of protein kinase function, but may also give insight into disease-linked mutations in other protein kinases such as the Parkinson's diseasecausative protein kinase Leucine-Rich Repeat Kinase (LRRK2). The LRRK2 gene was found mutated in families segregating with a form of familial parkinsonism (PARK8) (29). One of the earliest identified parkinsonism-associated mutations was the G2019S mutation, located within 
the DFG motif of the LRRK2 kinase domain (DYG in LRRK2) (30). The G2019S mutation is the most prevalent LRRK2 mutation found in PARK8 kindreds, and several studies have also documented association with sporadic Parkinson's disease (reviewed in (31)).

Located within the activation segment of the LRRK2 kinase domain, the G2019S mutation is expected to influence the regulation of LRRK2 kinase activity. This was confirmed by reports of enhanced autophosphorylation and substrate phosphorylation caused by the G2019S mutation in full length GFP-tagged LRRK2 immunoprecipitated from COS7 cells, and in affinity-purified myc-tagged LRRK2 expressed in HEK293 cells (32). How the G2019S mutation activates LRRK2 remains unclear. In G2019S mutant LRRK2, the glycine of the DFG motif is replaced by a serine residue. Activation of LRRK2 kinase activity by the mutation may be due to loss of some regulatory property conferred by the glycine, or gain of some interaction mediated by the serine side chain. In support of the first alternative, glycine is a unique residue and may carry out a unique function in the A-loop. Glycine lacks a side chain, and this allows rotation around the axes of the backbone $\mathrm{N}-\mathrm{C} \alpha$ and $\mathrm{C} \alpha-\mathrm{C}$ bonds to adopt angles not commonly seen in other residues. This flexibility is likely to be important for DFG-flip in catalysis (Fig. 3B). Indeed, in the imatinib-stabilized DFG-aspartate-out conformation of c-Abl, the glycine phi and psi angles are conventional, and could also occur for a serine at that position (33). However, in an inactive structure stabilized by the anilinoquinazolines derivative AZD0530, the glycine backbone adopts a conformation unique to glycine (34). This suggests that serine substitution at G2019 may act by constraining the flexibility usually conferred by glycine, limiting the range of available conformations. In this way, turnover rate of LRRK2 may depend on the unique range of motion of glycine at position 2019, which is lost upon mutation to serine. 
The observation that mutation of the glycine in the DFG motif induces activation is unique to LRRK2. For the second Parkinson's disease-associated protein kinase PINK1, mutation of the homologous glycine to alanine leads to a significant decrease in kinase activity (35). It is possible that replacement of the DFG glycine to a bulkier alanine inactivates PINK1 by limiting the DFG flip of the PINK1 mutant.

As DFG motif is involved in regulation of protein kinases, it is highly conserved. Kornov et al. (10) suggested that the lack of side chain of the DFG glycine allows the DFG motif to move between the inactive and inactive configuration without steric hindrance. Furthermore, the lack of side chain of the DFG glycine may also allow the DFG flip to occur rapidly without steric hindrance during catalysis. In light of this, it is intriguing that the Mnk2 kinase sequence contains a DFD motif rather a DFG motif. The crystal structure of the Mnk2 kinase domain reveals that it adopts a DFG-Asp-out conformation, further supporting the suggestion that the DFG glycine plays an important role in regulating the DFG-flip.

It is noteworthy that the pseudokinase CASK lacks the $\mathrm{Mg}^{++}$-chelating Asp in its DFG motif (the DFG aspartate is replaced by a glycine) and yet it is capable of catalyzing $\mathrm{Mg}^{2+}$ independent protein phosphorylation reactions (36). The crystal structure of CASK kinase domain revealed that it can bind the ATP analog, AMPPNP in a $\mathrm{Mg}^{++}$-independent manner. Identifying the DFG-equivalent motif capable of binding ATP and releasing ADP in catalysis will provide important insights into the regulation of CASK. It is likely that this putative DFGequivalent ATP-binding motif undergoes rapid configurational changes to facilitate ATP binding and ADP release in catalysis. 
Implications for other protein kinases II - effects of mutations of the gatekeeper residue on kinase activity and regulation

Replacement of the conserved gatekeeper threonine with isoleucine leads to activation of several tyrosine kinases (14) because the hydrophobic isoleucine likely enhances the hydrophobic spine assembly. In light of this, it will be of interest to ascertain if replacement of the gatekeeper residue with a hydrophobic amino acid residue will also activate other protein kinases such as the serine/threonine kinases and dual-specificity kinases is warranted. Results of the study will benefit the development of a universal approach to generate constitutively active mutants for studying the functions of protein kinases.

\section{Implications to the design of SFK inhibitors}

Owing to the significant role played by SFKs in cancer, much effort has been made to develop specific SFK inhibitors as therapeutics for cancer treatment. Although dasatinib, one of the SFK inhibitors has been successfully used in treating a subset of chronic myelogenous leukaemia patients who are resistant to the BCR-ABL inhibitor imatinib, all SFK inhibitors including in them dasatinib lack specificity - i.e. they can potentially induce significant side effects in patients by inhibiting other protein kinases and non-kinase proteins. Thus, lack of inhibitor selectivity was listed by Zhang et al. in their review as one of the major challenges in the field of protein kinase inhibitor development.(37).

Based upon the conformations adopted by protein kinases upon binding to inhibitors, researchers groups all known active site-targeting inhibitors into two types. Type 1 inhibitors recognize the active site of kinases in the DFG-Asp-in conformation, while the type 2 inhibitors bind the active site of kinases in the DFG-Asp-out conformation. As shown in Fig. 2 and 3, the 
DFG-Asp-in conformation is the only conformation capable of binding and properly aligning $\mathrm{Mg}^{2+}$-ATP and protein substrates for the phosphotransfer reaction. It is surprising that the DFGAsp-in conformations of individual protein kinases are very similar. Thus, type 1 inhibitors are expected to lack specificity. For example, dasatinib, a type 1 SFK inhibitor, also efficiently inhibit wide spectrum of protein kinases such as BCR-Abl, LIM domain kinase 2 and CSK (38). In contrast, there are much more structural variations among the DFG-Asp-out conformations of protein kinases. Type 2 inhibitors recognizing these conformations are expected to be much more specific for the individual kinases. Indeed, imatinib, a type 2 inhibitor of BCR-ABL exhibits a very narrow spectrum of kinase targets (38). Thus, it anticipated that more specific SFK inhibitors can be developed by refining the structures of the known type 2 inhibitors of SFKs $(19,20)$.

A third type of protein kinase inhibitors listed in the review by Zhang et al. are the allosteric inhibitors (37). These inhibits bind to regions outside the ATP-binding site of protein kinases. Since these binding sites are unique to individual kinases, the allosteric inhibitors are highly specific. For example, GNF2, a BCR-ABL inhibitor, specifically binds the myristoyl pocket in the large lobe of the Abl kinase domain. The myristoyl pocket is a unique allosteric site of the Abl kinase domain. In c-ABL, binding of the $\mathrm{N}$-terminal myristoyl group to this pocket underpins autoinhibition (39). Since BCR-ABL lacks the N-terminal myristoyl group, it is constitutively active and its myristoyl pocket is unoccupied. By binding to this unoccupied pocket, GNF-2 allosterically inhibits BCR-ABL but not c-ABL. The high degree of specificity of GNF-2 is illustrated by its ability to exert anti-proliferative effects exclusively to cells transformed by BCR-ABL. As shown in Fig. 6, the C-terminal tail of c-Src represents allosteric site to which CSK binds and allosterically induces the c-Src kinase domain to adopt a distinct 
inactive conformation (Fig. 6D). Given the high degree of sequence similarity of CSK and CHK (Fig. 6A), we predict that c-Src in the c-Src/CHK complex can also adopt this distinct inactive conformation. Small molecule compounds stabilizing c-Src in the distinct inactive conformation(s) found in the c-Src/CSK and c-Src/CHK complex are likely specific allosteric inhibitors of SFKs. These inhibitors are potential lead compounds for the development of therapeutic SFK inhibitors for the treatment of cancer. 


\section{Figure Legends}

Fig. 1 The structure of c-Src

A. The functional domains of SFKs. The N-terminal portion contains the fatty acid acylation motif to which myritoyl and/or palmitoyl moieties are added. A consensus autophosphorylation site $\left(\mathrm{Y}_{\mathrm{A}}\right)$ resides in the A-loop of the kinase domain. A consensus $\mathrm{C}$-terminal regulatory tyrosine $\left(\mathrm{Y}_{\mathrm{T}}\right)$ is specifically phosphorylated by CSK and CHK. B. The structure of the inactive conformation of the c-Src (PDB: 2SRC). The structure of truncated c-Src containing the SH3 and $\mathrm{SH} 2$ domains, the SH2-kinase linker (referred to as linker), the kinase domain and the C-terminal tail with the C-terminal regulatory tyrosine (Tyr-416, referred to as $\mathrm{Y}_{\mathrm{T}}$ ) phosphorylated. The kinase domain is trapped in the inactive conformation by two inhibitory intramolecular interactions: (i) the $\mathrm{pY}_{\mathrm{T}}$-SH2 interaction in which the phosphorylated $\mathrm{Y}_{\mathrm{T}}$ binds the $\mathrm{SH} 2$ domain, and (ii) the linker-SH3 interaction which involves binding of the SH2-kinase linker (linker) to the $\mathrm{SH} 3$ domain. In this configuration, the linker functions as a switch that governs the

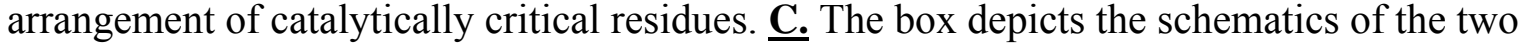
inhibitory intramolecular interactions.

Fig. 2 The hydrophobic spine in the active conformation organizes the catalytically critical residues appropriately for the phosphotransfer reaction.

A. The active conformation of c-Src kinase domain. The regulatory hydrophobic spine is made up of L325 of the $\beta 5$ strand, M314 of the C-helix, F405 of the activation loop and H384 of the catalytic loop. The gatekeeper residue (T338) makes intimate contact with L325 of the spine. Its mutation to isoleucine facilitates assembly of the hydrophobic spine. The linker is the most important regulatory motifs governing the assembly of the hydrophobic spine. Once assembled, the hydrophobic spine appropriately organizes the catalytically critical residues for the phosphorylation reaction. Proper organization of the catalytically critical residues involves the formation of a salt bridge between Glu-310 and Lys-295, the proper alignment of the catalytic base Asp-386 and the $\mathrm{Mg}^{2+}$-binding Asp404, displacement of the A-loop from the active site. The active conformation is stabilized by autophosphorylation at Tyr-416. This conformation is also called the DFG-Asp-in conformation as the Asp-404 is located inside the active site and participates in binding the $\mathrm{Mg}^{++}$ion. B. The inactive conformation of c-Src kinase domain. In this conformation, the hydrophobic spine is disassembled. Consequently, the catalytically critical residues are inappropriately aligned to prevent ATP binding. Furthermore, the A-loop is organized in such a way that Tyr-416 blocks entry of substrate protein to the active site. $\underline{\text { C. The }}$ DFG-Asp-out conformation. The DFG motif can undergo flipping motion. In this conformation, Phe-404 side chain is pointing towards the active site. Consequently, the Asp-404 side chain is pointing away from the active site. In the model of the catalytic cycle of SFKs, catalysis of the phosphorylation reaction requires reversible transition of the kinase domain between the DFGAsp-in and DFG-Asp-out conformations. The cartoons are schematics depicting assembly and disassembly of the hydrophobic spine in active and inactive conformations as well as alteration of the Phe-405 position in the DFG-Asp-out conformation. Both the linker and the gatekeeper residue participate in controlling assembly and disassembly of the hydrophobic spine. 
Fig. 3 The coupling pattern of the linker, C-helix and A-loop governs the transition of SFK from the inactive to active conformations

A. A close-up diagram showing interaction between the linker with the kinase domain in the inactive conformation. The linker, C-helix and A-loop are coupled by hydrophobic interactions. Trp-260 (W260) of the linker inserts in between the $\beta 4$-strand and the C-helix and interacts with a group of hydrophobic residues (upper dotted circle). The $\mathrm{C}$-helix is coupled to the activation loop (A-loop) by the hydrophobic cluster formed by Phe-307 and Met-314 of the C-helix and Leu-407 of the A-loop. B. A close-up diagram showing interaction between the linker and the kinase domain in the active conformation (PDB: 3DQW). Alteration of the coupling pattern of the linker, C-helix and A-loop governs transition of the kinase domain from the inactive to active conformation. In the active conformation, Trp-260 of the linker does not interact with hydrophobic residues in the C-helix. Furthermore, the hydrophobic cluster formed by Phe-307, Met-314 and Leu-407 are disassembled. C. A bird's-eye view of the structure in panel A. The networks of interactions between Trp-260 with residues (L325, Y326 and V328) in the kinase domain are shown. D. A bird's-eye view of the structure in panel B. The networks of interactions between residues in the linker (D258, W260, R264) with residues in the kinase domain are shown. Note the interactions between R264 with E331 and P333 at the loop joining the $\beta 4$ - and $\beta 5$-strands ( $\beta 4.5$ loop); hydrophobic interactions between W260 to residues in the kinase domain that form a hydrophobic pocket; electrostatic interactions between Lys-315 of the C-helix and Asp-258 of the linker.

Fig. 4 The pathway of conformational transition leading to activation of SFK. The linker, $\mathrm{C}$-helix and A-loop are coupled in a specific pattern that stabilizes the inactive conformation of the kinase. Transient alteration of the coupling pattern allows the kinase domain to transit from the inactive to active conformation. Results of molecular dynamics simulation studies identify two intermediates $I_{1}$ and $I_{2}$ in the pathway of the conformational transition (7). Disruption of the two inhibitory intramolecular interactions by ligands of the $\mathrm{SH} 2$ and $\mathrm{SH} 3$ domains is expected to facilitate the conformational transition to the active conformation. The active conformation of the kinase domain is further stabilized upon autophosphorylation of the conserved tyrosine $\left(\mathrm{Y}_{\mathrm{A}}\right.$, Tyr-416 of c-Src) in the A-loop. Although phosphorylation of the C-terminal regulatory tyrosine $\left(\mathrm{Y}_{\mathrm{T}}\right)$ by $\mathrm{CSK}$ and $\mathrm{CHK}$ and dissociation of the ligands of the $\mathrm{SH} 2$ and $\mathrm{SH} 3$ domains are involved in the conversion of the active conformation of the kinase domain to the inactive conformation, the conformational transition pathway has not been mapped.

\section{Fig. 5 Conformational transition of c-Src in catalysis}

The catalytic cycle of the SFK kinase domain involves reversible transition between the DFGAsp-in and DFG-Asp-out conformations. The catalytic conformational transition, termed DFG flip is coupled with protonation and deprotonation of the side chain of Asp-404. The DFG-flip involves flipping of Asp-404 and Phe-405 in and out of the active site. In the DFG-Asp-in conformation, the Asp-404 side chain is deprotonated and negatively charged, allowing it to chelate the ATP-binding $\mathrm{Mg}^{++}$. Phe-405, however, interacts with a group of hydrophobic residues. As all catalytically critical residues are properly aligned in the DFG-Asp-in conformation, the kinase domain efficiently catalyses phosphorylation of the protein substrate. 
To facilitate the release of $\mathrm{Mg}^{++}$-ADP, Asp-404 and Phe-405 exchange positions. The flip involves (i) Phe-405 moving out of the hydrophobic pocket and entering into the active site, and (ii) Asp-404 moving out of the active site and entering into the hydrophobic pocket. Asp-404 needs to lose its negative charge by protonation before it enters into the hydrophobic pocket. As the protonated Asp-404 side chain is unable to chelate $\mathrm{Mg}^{++}$-ADP, $\mathrm{Mg}^{++}$-ADP is released from the kinase. During transition from the DFG-Asp-out to the DFG-Asp-in conformations, Asp-404 is deprotonated. It is likely that the deprotonated Asp-404 side chain picks up a $\mathrm{Mg}^{++}$-ATP complex before it enters into the active site. The two boxes at the bottom of the diagram depict the arrangement of the linker, the gatekeeper residue and the hydrophobic spine residues in the DFG-Asp-in and DFG-Asp-out conformations (PDB: 3EL8).

\section{Fig. 6 Structure of c-Src/CSK complex}

A. Sequences of Src-binding motifs of CSK and the homologous regions in CHK. R279, R281 and R283 of $\alpha \mathrm{D}$-helix and R384 and R389 of $\alpha \mathrm{F}-\alpha \mathrm{G}$ loop of CSK and their homologs in CHK are underlined and labeled in red. B. The structure of c-Src/CSK complex (3D7U). The motifs in $\mathrm{c}$-Src and CSK involved in complex formation are labeled and the interacting residues are shown. C. The close-up view from the bottom of panel B - acidic residues in the C-terminal tail of c-Src (labeled in red) including D504, E510, E517 and D518 interact electrostatically with the cluster of basic residues residing in or near the $\alpha \mathrm{D}-\mathrm{helix}$ and $\alpha \mathrm{F}-\alpha \mathrm{G}$ loop in CSK kinase domain. D. c-Src in the c-Src/CSK complex adopts a distinct conformation. The disposition of key regulatory residues and motifs including W260 in the N-terminal segment (orange), E310 in the C-helix (green) and K295 in the $\beta 3$ strand are shown. In the active conformation, K295 interacts with $\alpha$ - and $\beta$-phosphates of $\mathrm{Mg}^{2+}$-ATP $\gamma$ S.

\section{ACKNOWLEDGEMENTS}

The authors' works described in this manuscript were supported by research grants of the

National Health and Medical Research Council of Australia, Cancer Council Victoria and

Australian Brain Foundation. Ryan Mills is a recipient of the Dowd Neuroscience Postgraduate

Research Fellowship. 


\section{References}

1. Martin GS. The hunting of the Src. Nat Rev Mol Cell Biol 2001; 2:467-75.

2. Ban K, Gao Y, Amin HM, et al. BCR-ABL1 mediates up-regulation of Fyn in chronic myelogenous leukemia. Blood 2008; 111:2904-8.

3. O'Hare T, Eide CA, Deininger MW. Persistent LYN signaling in imatinib-resistant, BCR-ABLindependent chronic myelogenous leukemia. J Natl Cancer Inst 2008; 100:908-9.

4. Hanna Z, Weng X, Kay DG, Poudrier J, Lowell C, Jolicoeur P. The pathogenicity of human immunodeficiency virus (HIV) type 1 Nef in CD4C/HIV transgenic mice is abolished by mutation of its SH3-binding domain, and disease development is delayed in the absence of Hck. J Virol 2001; 75:937892.

5. Sicheri F, Moarefi I, Kuriyan J. Crystal structure of the Src family tyrosine kinase Hck. Nature 1997; 385:602-9.

6. Xu W, Harrison SC, Eck MJ. Three-dimensional structure of the tyrosine kinase c-Src. Nature 1997; 385:595-602.

7. Yang S, Banavali NK, Roux B. Mapping the conformational transition in Src activation by cumulating the information from multiple molecular dynamics trajectories. Proc Natl Acad Sci U S A 2009; 106:3776-81.

8. Yang S, Roux B. Src kinase conformational activation: thermodynamics, pathways, and mechanisms. PLoS Comput Biol 2008; 4:e1000047.

9. Adams JA. Kinetic and Catalytic Mechanisms of Protein Kinases. Chem. Rev. 2001; 101:2271-90.

10. Kornev AP, Haste NM, Taylor SS, Eyck LF. Surface comparison of active and inactive protein kinases identifies a conserved activation mechanism. Proc Natl Acad Sci U S A 2006; 103:17783-8.

11. Emrick MA, Lee T, Starkey PJ, Mumby MC, Resing KA, Ahn NG. The gatekeeper residue controls autoactivation of ERK2 via a pathway of intramolecular connectivity. Proc Natl Acad Sci U S A 2006; 103:18101-6.

12. Ten Eyck LF, Taylor SS, Kornev AP. Conserved spatial patterns across the protein kinase family. Biochim Biophys Acta 2008; 1784:238-43.

13. Bukhtiyarova M, Karpusas M, Northrop K, Namboodiri HV, Springman EB. Mutagenesis of p38alpha MAP kinase establishes key roles of Phe169 in function and structural dynamics and reveals a novel DFG-OUT state. Biochemistry 2007; 46:5687-96.

14. Azam M, Seeliger MA, Gray NS, Kuriyan J, Daley GQ. Activation of tyrosine kinases by mutation of the gatekeeper threonine. Nat Struct Mol Biol 2008; 15:1109-18.

15. Huang $\mathrm{K}$, Wang $\mathrm{YH}, \mathrm{Brown} \mathrm{A}$, Sun $\mathrm{G}$. Identification of $\mathrm{N}$-terminal lobe motifs that determine the kinase activity of the catalytic domains and regulatory strategies of Src and Csk protein tyrosine kinases. J Mol Biol 2009; 386:1066-77.

16. Lerner EC, Smithgall TE. SH3-dependent stimulation of Src-family kinase autophosphorylation without tail release from the SH2 domain in vivo. Nat Struct Biol 2002; 9:365-9.

17. Lerner EC, Trible RP, Schiavone AP, Hochrein JM, Engen JR, Smithgall TE. Activation of the SrC family kinase Hck without SH3-linker release. J Biol Chem 2005; 280:40832-7.

18. Cowan-Jacob SW, Fendrich G, Manley PW, et al. The crystal structure of a c-Src complex in an active conformation suggests possible steps in c-Src activation. Structure 2005; 13:861-71.

19. Seeliger MA, Ranjitkar P, Kasap C, et al. Equally potent inhibition of c-Src and Abl by compounds that recognize inactive kinase conformations. Cancer Res 2009; 69:2384-92. 
20. Dar AC, Lopez, M.S., and Shokat, K.M. Small Molecule Recognition o c-Src via the ImatinibBinding Conformation. Chem. \& Biol. 2008; 15:1015-22.

21. Shan $\mathrm{Y}$, Seeliger MA, Eastwood MP, et al. A conserved protonation-dependent switch controls drug binding in the Abl kinase. Proc Natl Acad Sci U S A 2009; 106:139-44.

22. Chong YP, la KK, Mulhern TD, Cheng HC. Endogenous and synthetic inhibitors of the Src-family protein tyrosine kinases. Biochim Biophys Acta 2005; 1754:210-20.

23. Chong YP, Chan AS, Chan KC, et al. C-terminal Src kinase-homologous kinase (CHK), a unique inhibitor inactivating multiple active conformations of Src family tyrosine kinases. J Biol Chem 2006; 281:32988-99.

24. Chong YP, Mulhern TD, Zhu HJ, et al. A novel non-catalytic mechanism employed by the Cterminal Src-homologous kinase to inhibit Src-family kinase activity. J Biol Chem 2004; 279:20752-66. 25. Sondhi D, Xu W, Songyang Z, Eck MJ, Cole PA. Peptide and protein phosphorylation by protein tyrosine kinase Csk: insights into specificity and mechanism. Biochemistry 1998; 37:165-72.

26. Lee S, Lin X, Nam NH, Parang K, Sun G. Determination of the substrate-docking site of protein tyrosine kinase C-terminal Src kinase. Proc Natl Acad Sci U S A 2003; 100:14707-12.

27. Lee $S$, Ayrapetov MK, Kemble DJ, Parang K, Sun G. Docking-based substrate recognition by the catalytic domain of a protein tyrosine kinase, C-terminal Src kinase (Csk). J Biol Chem 2006; 281:8183-9. 28. Levinson NM, Seeliger MA, Cole PA, Kuriyan J. Structural basis for the recognition of c-Src by its inactivator Csk. Cell 2008; 134:124-34.

29. Paisan-Ruiz C, Jain S, Evans EW, et al. Cloning of the gene containing mutations that cause PARK8-linked Parkinson's disease. Neuron 2004; 44:595-600.

30. Hernandez DG, Paisan-Ruiz C, Mclnerney-Leo A, et al. Clinical and positron emission tomography of Parkinson's disease caused by LRRK2. Ann Neurol 2005; 57:453-6.

31. Belin AC, Westerlund M. Parkinson's disease: a genetic perspective. FEBS J 2008; 275:1377-83.

32. Greggio $E$, Jain $S$, Kingsbury $A$, et al. Kinase activity is required for the toxic effects of mutant LRRK2/dardarin. Neurobiol Dis 2006; 23:329-41.

33. Seeliger MA, Nagar B, Frank F, Cao X, Henderson MN, Kuriyan J. c-Src binds to the cancer drug imatinib with an inactive Abl/c-Kit conformation and a distributed thermodynamic penalty. Structure 2007; 15:299-311.

34. Hennequin LF, Ballard P, Boyle FT, et al. Novel 4-anilinoquinazolines with C-6 carbon-linked side chains: synthesis and structure-activity relationship of a series of potent, orally active, EGF receptor tyrosine kinase inhibitors. Bioorg Med Chem Lett 2006; 16:2672-6.

35. Sim CH, Lio DS, Mok SS, et al. C-terminal truncation and Parkinson's disease-associated mutations down-regulate the protein serine/threonine kinase activity of PTEN-induced kinase-1. Hum Mol Genet 2006; 15:3251-62.

36. Mukherjee $\mathrm{K}$, Sharma M, Urlaub H, et al. CASK Functions as a Mg2+-independent neurexin kinase. Cell 2008; 133:328-39.

37. Zhang J, Yang PL, Gray NS. Targeting cancer with small molecule kinase inhibitors. Nat Rev Cancer 2009; 9:28-39.

38. Rix U, Hantschel O, Durnberger G, et al. Chemical proteomic profiles of the BCR-ABL inhibitors imatinib, nilotinib, and dasatinib reveal novel kinase and nonkinase targets. Blood 2007; 110:4055-63. 39. Nagar B, Hantschel O, Young MA, et al. Structural basis for the autoinhibition of c-Abl tyrosine kinase. Cell 2003; 112:859-71. 
A.

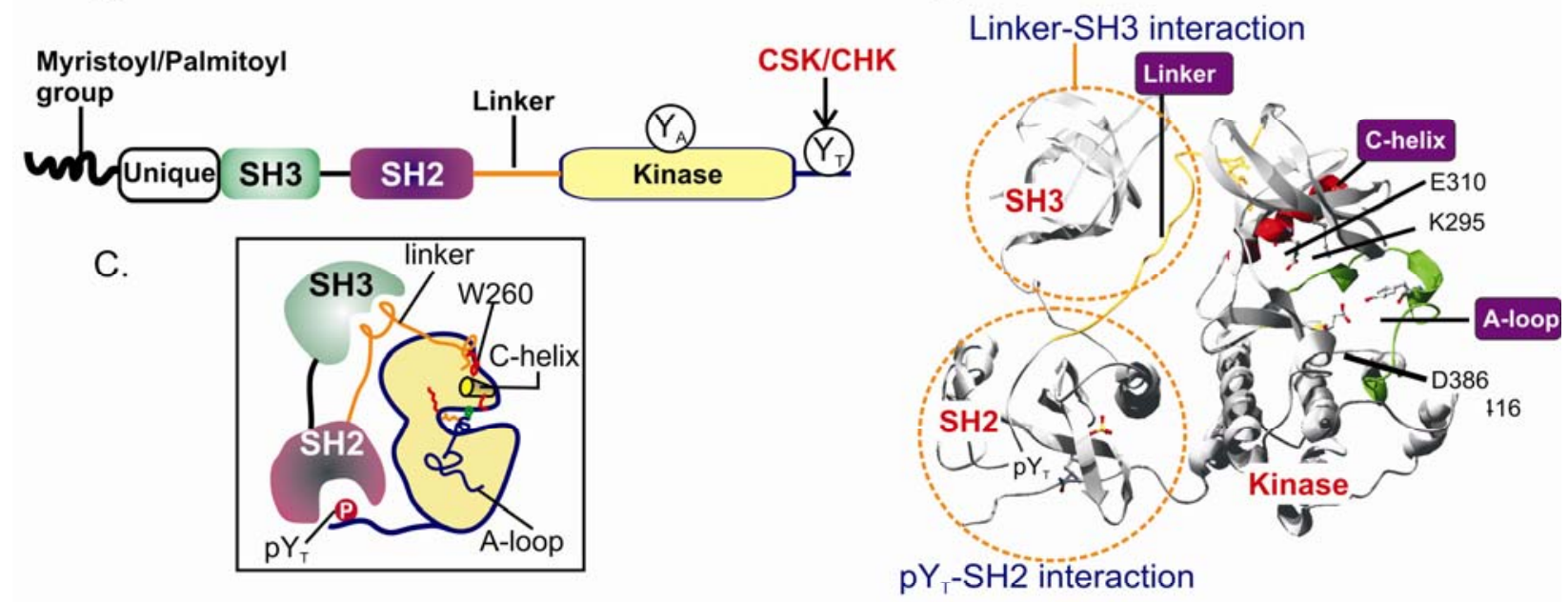

Fig. 1

\section{Fig. 1 The structure of c-Src}

A. The functional domains of SFKs. The N-terminal portion contains the fatty acid acylation motif to which myritoyl and/or palmitoyl moieties are added. A consensus autophosphorylation site $\left(\mathrm{Y}_{\mathrm{A}}\right)$ resides in the A-loop of the kinase domain. A consensus C-terminal regulatory tyrosine $\left(\mathrm{Y}_{\mathrm{T}}\right)$ is specifically phosphorylated by CSK and CHK. B. The structure of the inactive conformation of the c-Src (PDB: 2SRC). The structure of truncated c-Src containing the SH3 and SH2 domains, the SH2-kinase linker (referred to as linker), the kinase domain and the $\mathrm{C}$-terminal tail with the $\mathrm{C}$-terminal regulatory tyrosine (Tyr-416, referred to as $\mathrm{Y}_{\mathrm{T}}$ ) phosphorylated. The kinase domain is trapped in the inactive conformation by two inhibitory intramolecular interactions: (i) the $\mathrm{pY}_{\mathrm{T}}-\mathrm{SH} 2$ interaction in which the phosphorylated $\mathrm{Y}_{\mathrm{T}}$ binds the SH2 domain, and (ii) the linker-SH3 interaction which involves binding of the SH2-kinase linker (linker) to the SH3 domain. In this configuration, the linker functions as a switch that governs the

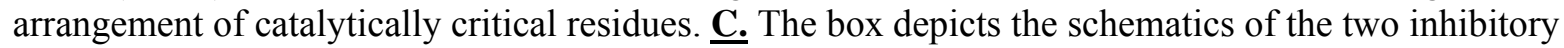
intramolecular interactions. 
A.
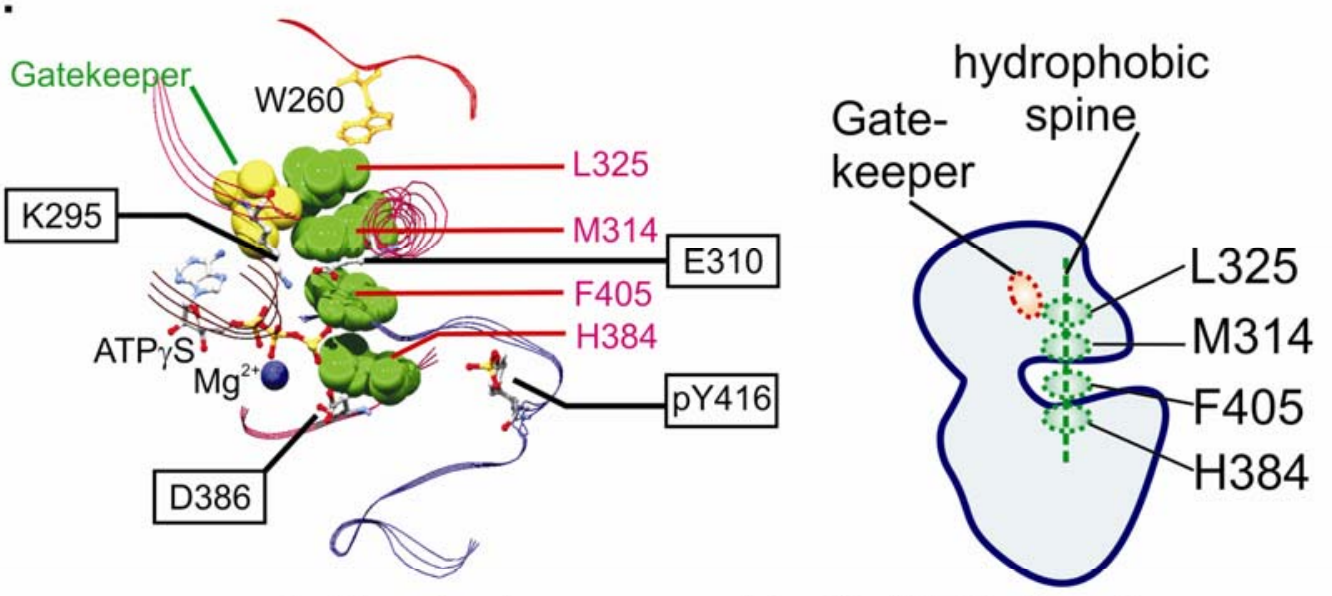

Active (spine assembled), DFG-Asp-in

B.

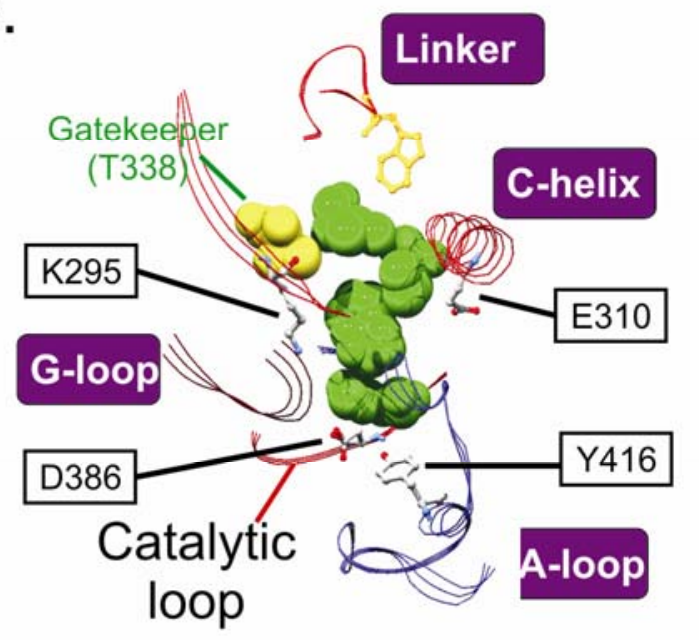

Inactive (spine disassembled)

C.

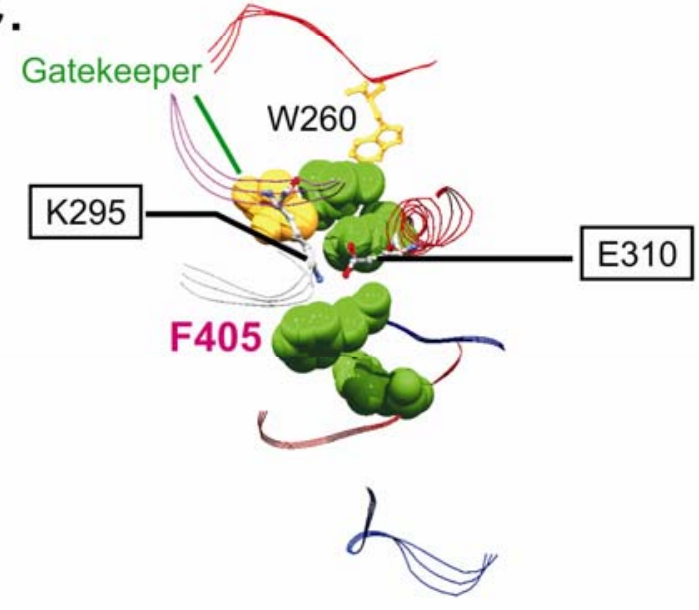

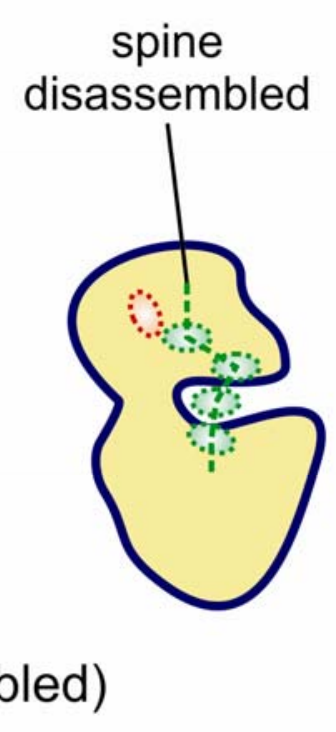

Fig. 2

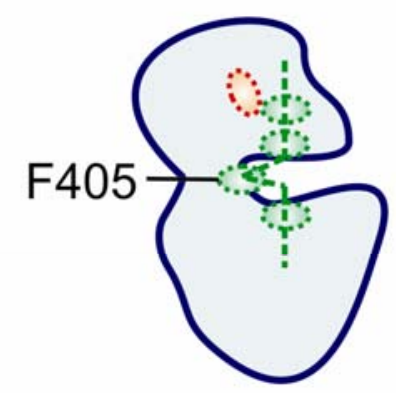


Fig. 2 The hydrophobic spine in the active conformation organizes the catalytically critical residues appropriately for the phosphotransfer reaction.

A. The active conformation of c-Src kinase domain. The regulatory hydrophobic spine is made up of L325 of the $\beta 5$ strand, M314 of the C-helix, F405 of the activation loop and H384 of the catalytic loop. The gatekeeper residue (T338) makes intimate contact with L325 of the spine. Its mutation to isoleucine facilitates assembly of the hydrophobic spine. The linker is the most important regulatory motifs governing the assembly of the hydrophobic spine. Once assembled, the hydrophobic spine appropriately organizes the catalytically critical residues for the phosphorylation reaction. Proper organization of the catalytically critical residues involves the formation of a salt bridge between Glu-310 and Lys-295, the proper alignment of the catalytic base Asp-386 and the $\mathrm{Mg}^{2+}$-binding Asp404, displacement of the A-loop from the active site. The active conformation is stabilized by autophosphorylation at Tyr-416. This conformation is also called the DFG-Asp-in conformation as the Asp-404 is located inside the active site and participates in binding the $\mathrm{Mg}^{++}$ion. B. The inactive conformation of c-Src kinase domain. In this conformation, the hydrophobic spine is disassembled. Consequently, the catalytically critical residues are inappropriately aligned to prevent ATP binding. Furthermore, the A-loop is organized in such a way that Tyr-416 blocks entry of substrate protein to the active site. $\mathbf{C}$. The DFG-Asp-out conformation. The DFG motif can undergo flipping motion. In this conformation, Phe-404 side chain is pointing towards the active site. Consequently, the Asp-404 side chain is pointing away from the active site. In the model of the catalytic cycle of SFKs, catalysis of the phosphorylation reaction requires reversible transition of the kinase domain between the DFGAsp-in and DFG-Asp-out conformations. The cartoons are schematics depicting assembly and disassembly of the hydrophobic spine in active and inactive conformations as well as alteration of the Phe-405 position in the DFG-Asp-out conformation. Both the linker and the gatekeeper residue participate in controlling assembly and disassembly of the hydrophobic spine. 

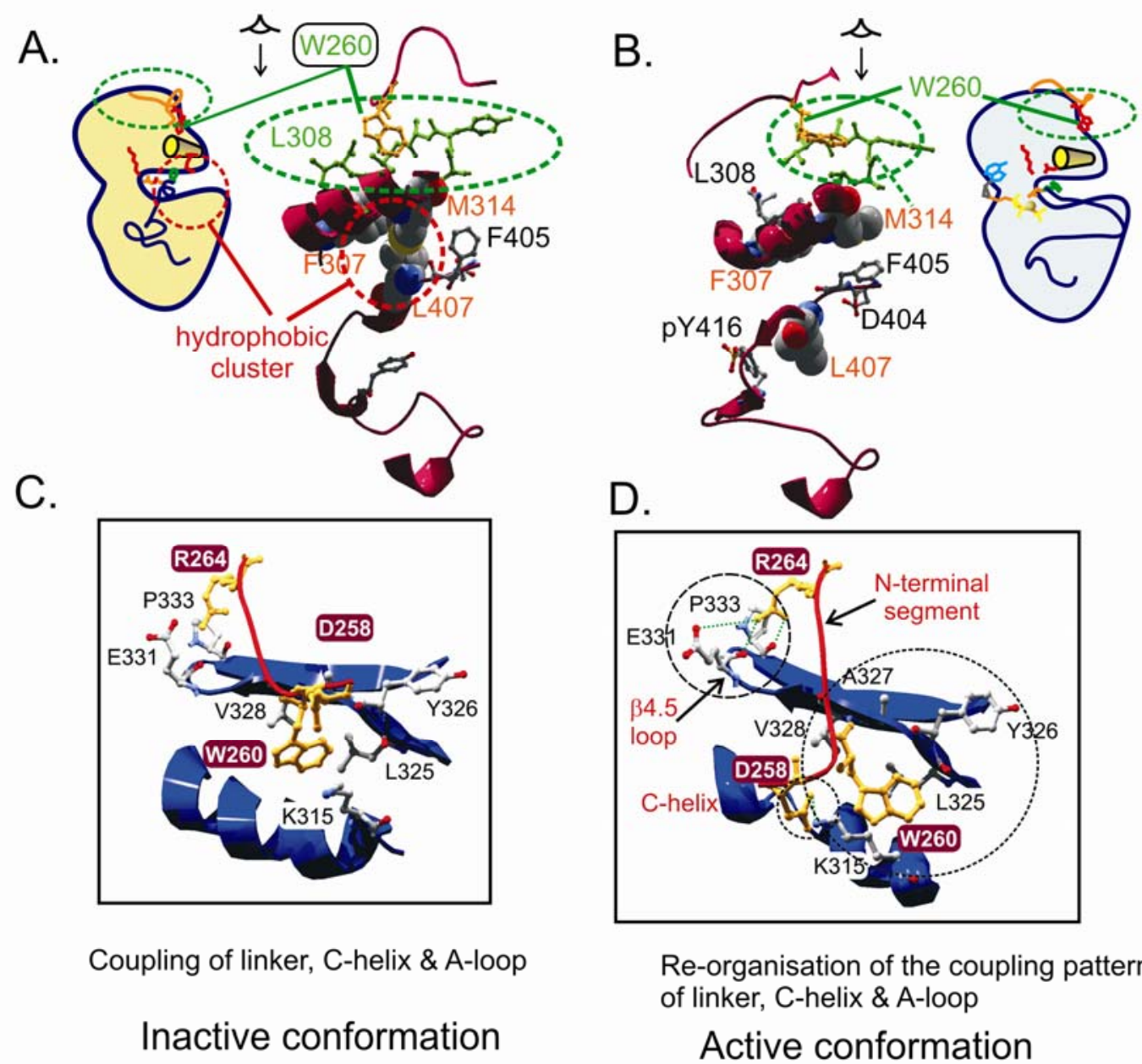

Re-organisation of the coupling pattern of linker, C-helix \& A-loop

Active conformation

Fig. 3

Fig. 3 The coupling pattern of the linker, C-helix and A-loop governs the transition of SFK from the inactive to active conformations

B. A close-up diagram showing interaction between the linker with the kinase domain in the inactive conformation. The linker, C-helix and A-loop are coupled by hydrophobic interactions. Trp-260 (W260) of the linker inserts in between the $\beta 4$-strand and the C-helix and interacts with a group of hydrophobic residues (upper dotted circle). The $\mathrm{C}$-helix is coupled to the activation loop (A-loop) by the hydrophobic cluster formed by Phe-307 and Met-314 of the C-helix and Leu-407 of the A-loop. B. A close-up diagram showing interaction between the linker and the kinase domain in the active conformation (PDB: 3DQW). Alteration of the coupling pattern of the linker, C-helix and A-loop governs transition of the kinase domain from the inactive to active conformation. In the active conformation, Trp-260 of the linker does not interact with hydrophobic residues in the C-helix. Furthermore, the hydrophobic cluster formed by Phe-307, Met-314 and Leu-407 are disassembled. C. A bird's-eye view of the structure in panel A. The networks of interactions between Trp-260 with residues (L325, Y326 and V328) in the kinase

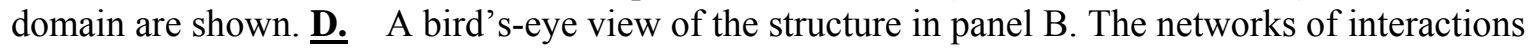
between residues in the linker (D258, W260, R264) with residues in the kinase domain are shown. Note the interactions between R264 with E331 and P333 at the loop joining the $\beta 4$ - and $\beta 5$-strands ( $\beta 4.5$ loop); hydrophobic interactions between W260 to residues in the kinase domain that form a hydrophobic pocket; electrostatic interactions between Lys-315 of the C-helix and Asp-258 of the linker. 


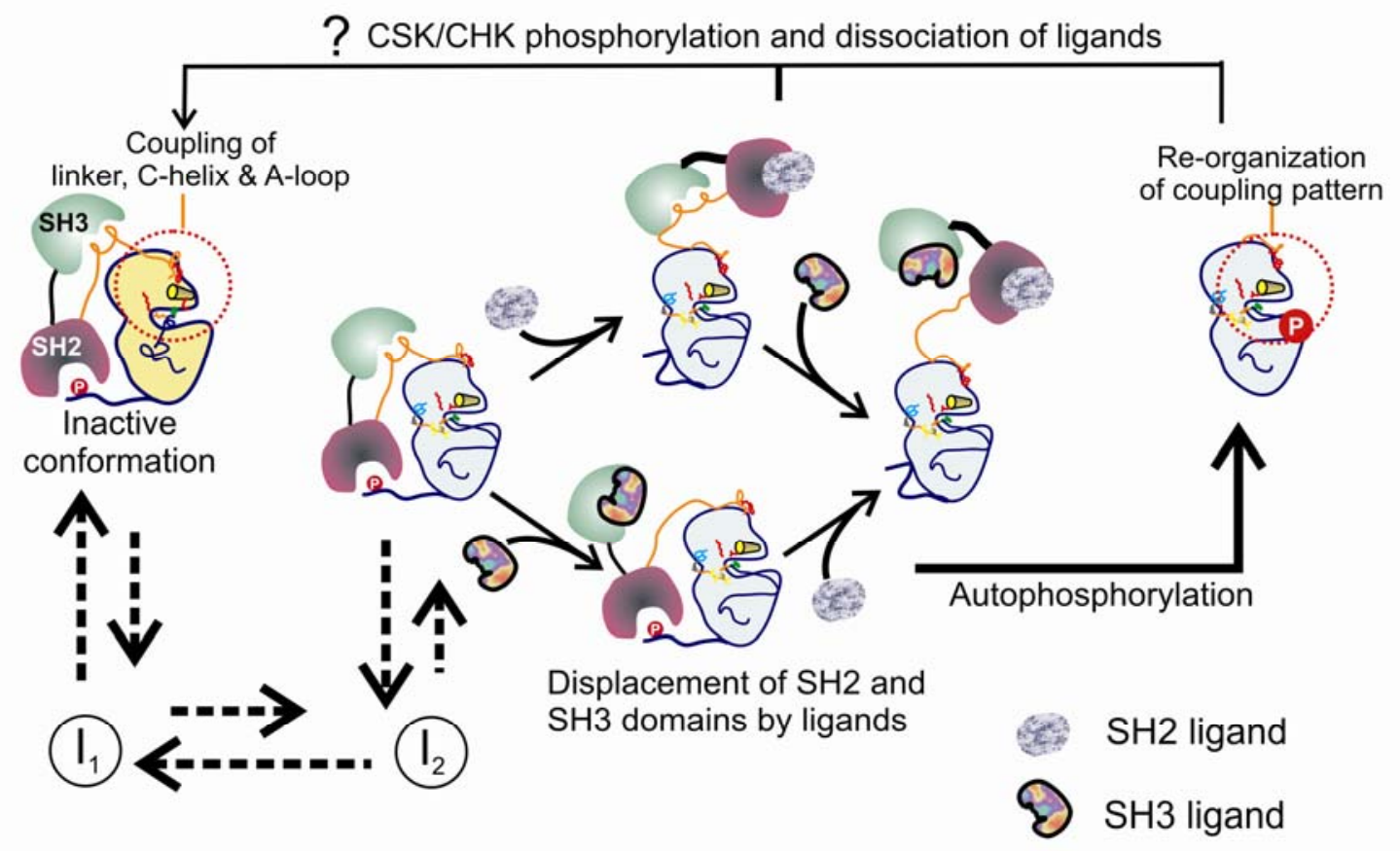

Fig. 4

Fig. 4 The pathway of conformational transition leading to activation of SFK. The linker, $\mathrm{C}$-helix and A-loop are coupled in a specific pattern that stabilizes the inactive conformation of the kinase. Transient alteration of the coupling pattern allows the kinase domain to transit from the inactive to active conformation. Results of molecular dynamics simulation studies identify two intermediates $I_{1}$ and $I_{2}$ in the pathway of the conformational transition (7). Disruption of the two inhibitory intramolecular interactions by ligands of the $\mathrm{SH} 2$ and $\mathrm{SH} 3$ domains is expected to facilitate the conformational transition to the active conformation. The active conformation of the kinase domain is further stabilized upon autophosphorylation of the conserved tyrosine $\left(\mathrm{Y}_{\mathrm{A}}\right.$, Tyr-416 of c-Src) in the A-loop. Although phosphorylation of the C-terminal regulatory tyrosine $\left(\mathrm{Y}_{\mathrm{T}}\right)$ by $\mathrm{CSK}$ and $\mathrm{CHK}$ and dissociation of the ligands of the $\mathrm{SH} 2$ and $\mathrm{SH} 3$ domains are involved in the conversion of the active conformation of the kinase domain to the inactive conformation, the conformational transition pathway has not been mapped. 


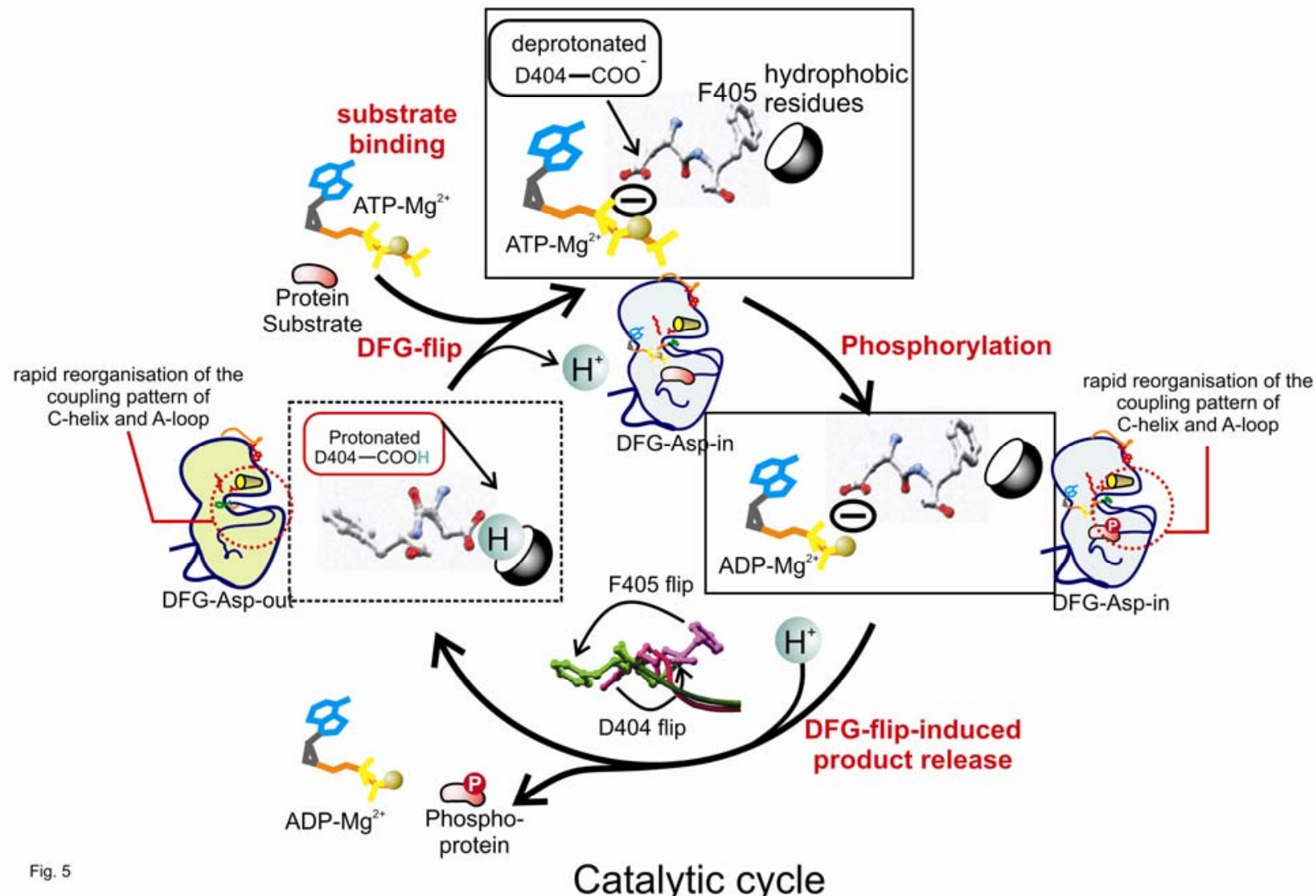

Fig. 5 Conformational transition of c-Src in catalysis

The catalytic cycle of the SFK kinase domain involves reversible transition between the DFGAsp-in and DFG-Asp-out conformations. The catalytic conformational transition, termed DFG flip is coupled with protonation and deprotonation of the side chain of Asp-404. The DFG-flip involves flipping of Asp-404 and Phe-405 in and out of the active site. In the DFG-Asp-in conformation, the Asp-404 side chain is deprotonated and negatively charged, allowing it to chelate the ATP-binding $\mathrm{Mg}^{++}$. Phe-405, however, interacts with a group of hydrophobic residues. As all catalytically critical residues are properly aligned in the DFG-Asp-in conformation, the kinase domain efficiently catalyses phosphorylation of the protein substrate. To facilitate the release of $\mathrm{Mg}^{++}$-ADP, Asp-404 and Phe-405 exchange positions. The flip involves (i) Phe-405 moving out of the hydrophobic pocket and entering into the active site, and (ii) Asp-404 moving out of the active site and entering into the hydrophobic pocket. Asp-404 needs to lose its negative charge by protonation before it enters into the hydrophobic pocket. As the protonated Asp-404 side chain is unable to chelate $\mathrm{Mg}^{++}$-ADP, $\mathrm{Mg}^{++}$-ADP is released from the kinase. During transition from the DFG-Asp-out to the DFG-Asp-in conformations, Asp-404 is deprotonated. It is likely that the deprotonated Asp-404 side chain picks up a $\mathrm{Mg}^{++}$-ATP complex before it enters into the active site. The two boxes at the bottom of the diagram depict the arrangement of the linker, the gatekeeper residue and the hydrophobic spine residues in the DFG-Asp-in and DFG-Asp-out conformations (PDB: 3EL8). 
A.

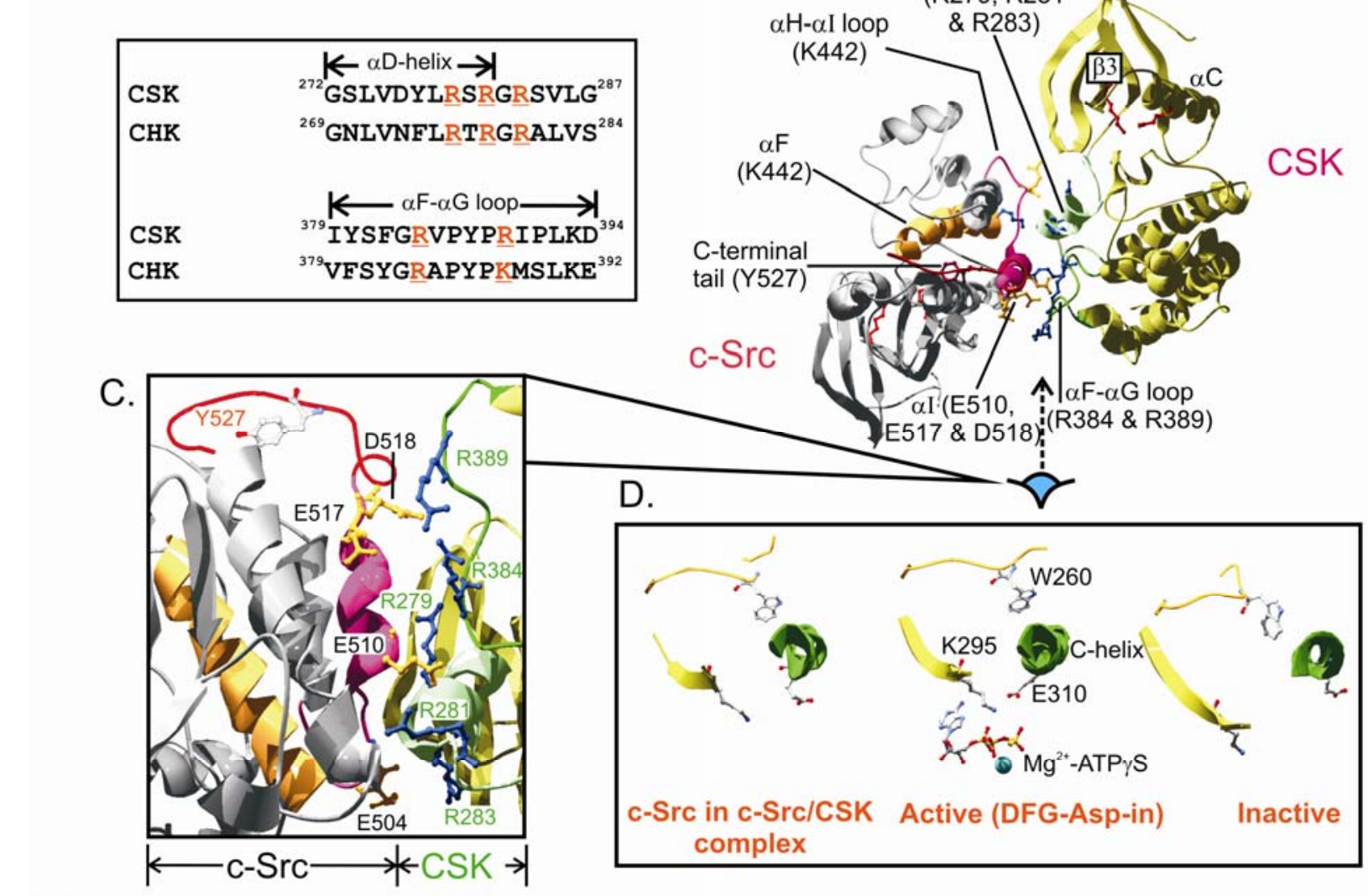

B. $\alpha \mathrm{D}$

Fig. 6

\section{Fig. 6 Structure of c-Src/CSK complex}

A. Sequences of Src-binding motifs of CSK and the homologous regions in CHK. R279, R281 and R283 of $\alpha \mathrm{D}$-helix and R384 and R389 of $\alpha \mathrm{F}-\alpha \mathrm{G}$ loop of CSK and their homologs in CHK are underlined and labeled in red. B. The structure of c-Src/CSK complex (3D7U). The motifs in $\mathrm{c}-\mathrm{Src}$ and CSK involved in complex formation are labeled and the interacting residues are shown. . The close-up view from the bottom of panel B - acidic residues in the C-terminal tail of c-Src (labeled in red) including D504, E510, E517 and D518 interact electrostatically with the cluster of basic residues residing in or near the $\alpha \mathrm{D}-$ helix and $\alpha \mathrm{F}-\alpha \mathrm{G}$ loop in CSK kinase domain. D. c-Src in the c-Src/CSK complex adopts a distinct conformation. The disposition of key regulatory residues and motifs including W260 in the N-terminal segment (orange), E310 in the C-helix (green) and K295 in the $\beta 3$ strand are shown. In the active conformation, K295 interacts with $\alpha$ - and $\beta$-phosphates of $\mathrm{Mg}^{2+}$-ATP $\gamma$ S. 